\title{
Localized PEPII Storage Ring Optics Measurements
}

\author{
Jonathan Landy \\ Office of Science, SULI Program \\ California Institute of Technology \\ Stanford Linear Accelerator Center \\ Menlo Park, California
}

August 19, 2005

Prepared in partial fulfillment of the requirements of the Office of Science, DOE Science Undergraduate Laboratory Internship (SULI) Program under the direction of Dr. Yiton Yan in the Accelerator Research Department A at Stanford Linear Accelerator Center.

Participant:

Signature

Research Advisor:

Signature 
Localized PEPII Storage Ring Optics Measurements. JONATHAN LANDY (California Institute of Technology, Pasadena, CA 91126) YITON YAN (Stanford Linear Accelerator Center, 2575 Sand Hill Road Menlo Park, CA 94025)

\begin{abstract}
The current technique employed to determine the parameters which specify the betatron oscillation in the PEPII ring at SLAC is a global procedure in that the data from each $B P M$ (Beam Position Monitor) is weighted equally. However for more accurate interaction point (IP) measurements it would be beneficial to weight the data from the $B P M \mathrm{~s}$ closest to the IP much more heavily. Researchers are thus considering the possibility of developing a technique to determine the oscillation parameters near the IP using as few $B P M$ s as possible. In this paper, allowing $B P M$ gains and cross coupling, we show analytically that given data from $N B P M$ s there remain $6 N+2$ degrees of freedom in the matrices $M_{A, A}$, $M_{B, A}, \ldots, M_{N, N-1}$ unspecified by the observable data alone. From this we demonstrate that data from at least $3 B P M \mathrm{~s}$ is required to completely specify the system when the transfer maps between $B P M \mathrm{~s}$ are assumed known, and that $4 B P M$ s may be more suitable.
\end{abstract}




\section{Contents}

1 Introduction 4

2 One-Dimensional Case

2.1 Single BPM Analysis . . . . . . . . . . . . . . . . 5

2.2 Multiple BPM Analysis . . . . . . . . . . . . . . . . . 10

2.3 Results in Terms of Standard Parameters . . . . . . . . . . . . 13

2.4 Approximate Solution . . . . . . . . . . . . . . . . . . . . 14

2.5 A Note on Rational Tunes . . . . . . . . . . . . . . . . . . 15

3 Two-Dimensional Case $\quad 15$

3.1 Single BPM Analysis . . . . . . . . . . . . . . . . . . . 15

3.2 Multiple BPM Analysis . . . . . . . . . . . . . . . . 20

3.3 Approximate Solution and Future Work . . . . . . . . . . . 22

4 Acknowledgements 23 


\section{Introduction}

As particles circle around a storage ring, such as the PEPII ring at SLAC, they move longitudinally in a tight beam. The particles will also have transverse momenta and therefore will tend to diverge. To prevent this from happening, in addition to the required bending dipoles, a series of quadrupole magnets is used to alternately focus the beam and then defocus the beam. As the particles move around the ring they will then oscillate in transverse phase space and are prevented from diverging. This oscillation is known as the betatron motion of the beam and is uniquely determined at each BPM (Beam Position Monitor) by 10 beam parameters. In this paper we will be investigating how many of these parameters can be determined from orbit data.

The transverse phase space position of the beam at $B P M_{A}$ and turn $n$ around the ring is denoted by $\overrightarrow{x_{n, A}}=\left(x, p_{x}, y, p_{y}\right)_{n, A}^{T}$, although we will sometimes drop the subscript specifying the $B P M$ when the meaning is clear. The position of the beam at $B P M_{A}$ uniquely determines what it will be when it reaches $B P M_{B}$. Therefore we can define a function $f_{B, A}$ as follows:

$$
f_{B, A}: \overrightarrow{x_{n, A}} \rightarrow \overrightarrow{x_{n, B}}
$$

Thus $f_{B, A}$ maps the beam's phase space position at $B P M_{A}$ to what it will be at $B P M_{B}$. In particular we can define the 1-turn map $f_{A, A}$ which maps the phase space position at $B P M_{A}$ at turn $n$ to what it will be at turn $n+1$ :

$$
f_{A, A}: \overrightarrow{x_{n, A}} \rightarrow \overrightarrow{x_{n+1, A}}
$$

These functions are known to be largely linear and therefore we may approximate these maps by $4 \times 4$ matrices which operate on the phase space. For example, the linear approximation of $f_{A, A}$ will be denoted by $M_{A, A}$. If it were possible to measure all components of the beam's phase space position as it passed by the $B P M$ it would be a simple matter to determine this matrix. We would only need to invert the orbit as follows:

$$
\begin{gathered}
M_{A, A}\left(\begin{array}{cccc}
x_{0} & x_{1} & x_{2} & x_{3} \\
p_{x_{0}} & p_{x_{1}} & p_{x_{2}} & p_{x_{3}} \\
y_{0} & y_{1} & y_{2} & y_{3} \\
p_{y_{0}} & p_{y_{1}} & p_{y_{2}} & p_{y_{3}}
\end{array}\right)_{A}=\left(\begin{array}{cccc}
x_{1} & x_{2} & x_{3} & x_{4} \\
p_{x_{1}} & p_{x_{2}} & p_{x_{3}} & p_{x_{4}} \\
y_{1} & y_{2} & y_{3} & y_{4} \\
p_{y_{1}} & p_{y_{2}} & p_{y_{3}} & p_{y_{4}}
\end{array}\right)_{A} \rightarrow \\
\left.M_{A, A}=\left(\begin{array}{ccccc}
x_{1} & x_{2} & x_{3} & x_{4} \\
p_{x_{1}} & p_{x_{2}} & p_{x_{3}} & p_{x_{4}} \\
y_{1} & y_{2} & y_{3} & y_{4} \\
p_{y_{1}} & p_{y_{2}} & p_{y_{3}} & p_{y_{4}}
\end{array}\right)_{A}\left(\begin{array}{cccc}
x_{0} & x_{1} & x_{2} & x_{3} \\
p_{x_{0}} & p_{x_{1}} & p_{x_{2}} & p_{x_{3}} \\
y_{0} & y_{1} & y_{2} & y_{3} \\
p_{y_{0}} & p_{y_{1}} & p_{y_{2}} & p_{y_{3}}
\end{array}\right)_{A}\right)_{A}
\end{gathered}
$$

Knowing the phase space position at 5 consecutive turns thus gives you complete information about the map. Further, the position of the beam at any later time is in the space spanned by the first 4 positions. This implies that no new information can be obtained by looking at more turns of the orbit. 
Unfortunately, the $B P M \mathrm{~s}$ can only measure the spatial coordinates of the beam and cannot measure its transverse momenta. Thus we are only able to measure half of the coordinates of its phase space position. For this reason it has been assumed that the information one can get from orbit data is insufficient to solve for the parameters without some a priori information about the machine lattice. We confirm this is true; however, it may be possible that some of the information lost about the map which is contained in the momenta at each turn could be recovered by looking at the $x$ and $y$ positions at later turns in the orbit. In this paper we demonstrate rigorously the number of parameters left unspecified by the orbit data and further give a lower bound on the number of $B P M \mathrm{~s}$ required to determine all the parameters when the matrices $M_{B, A}$, $M_{C, B}$, etc. are assumed known.

\section{One-Dimensional Case}

We will begin by analyzing lattices which oscillate only in the $x$-direction. In section 2.1 we will look at what can be determined from data from 1 BPM alone. In section 2.2 we will analyze the case of data from 2 or more BPMs. In section 2.3 we will discuss our results in terms of the machine parameters. And in section 2.4 we will demonstrate how a natural assumption permits a solution.

\subsection{Single BPM Analysis}

In this section we will prove that given the $x$-orbit data from a single $B P M, B P M_{A}$, it is impossible to determine the map $M_{A, A}$. Further we will show that there are in fact 2 degrees of freedom in $M_{A, A}$ left unspecified by this data alone.

\section{Symplectic Matrices and Normalized Phase Space}

A $2 \times 2$ symplectic matrix $M$ is defined as one such that:

$$
M^{T} S M=S, \quad \text { where } S=\left(\begin{array}{cc}
0 & 1 \\
-1 & 0
\end{array}\right)
$$

In the $2 \times 2$ case, it is easy to show that the symplectic condition is equivalent to the condition $\operatorname{det}(M)=1$.

One condition on the matrices $M_{A, A}, M_{B, A}$, etc. is that they be symplectic. This is a consequence of the fact these matrices represent systems in which the hamiltonian is time-independent. Another condition on $M_{A, A}$ is that its eigenvalues be complex-conjugates and of modulus 1 . We can see that is true as follows:

As $M_{A, A}$ is symplectic $\rightarrow \lambda_{1} \lambda_{2}=1$ where $\lambda_{1}, \lambda_{2}$ are its eigenvalues. As the machine is designed so that the orbits of $M_{A, A}$ will remain bounded it cannot be true that either $\left|\lambda_{1}\right|$ or $\left|\lambda_{2}\right|>1$, as this would result in an expanding orbit. This implies $\left|\lambda_{1}\right|=\left|\lambda_{2}\right|=1$. If $\lambda_{1}, \lambda_{2}$ are complex, then they are 
necessarily conjugate by the conjugate root theorem. Also if they are real, $\lambda_{1} \lambda_{2}=1 \rightarrow \lambda_{1}=\lambda_{2}$ and again they must be conjugate.

With these two conditions on $M_{A, A}$ it can be shown that it is always possible to decompose $M_{A, A}$ as follows:

$$
\begin{gathered}
M_{A, A}=A_{A} R_{A, A} A_{A}^{-1} \text { with, } \\
A_{A}=\left(\begin{array}{cc}
\sqrt{\beta_{A}} & 0 \\
\frac{-\alpha_{A}}{\sqrt{\beta_{A}}} & \frac{1}{\sqrt{\beta_{A}}}
\end{array}\right) \text { and } R_{A, A}=\left(\begin{array}{cc}
\cos \mu_{A} & \sin \mu_{A} \\
-\sin \mu_{A} & \cos \mu_{A}
\end{array}\right)
\end{gathered}
$$

Thus the matrix $A_{A}^{-1}$ transforms to a normalized space in which the one turn map is a simple clockwise rotation which induces circular orbits.

This decomposition explicitly shows that there are 3 parameters determining the matrix $M_{A, A}$ in the 1-Dimensional case. The rotation angle $\mu_{A}$ is set by the operators of the machine during runtime. This angle is always chosen so that the tune, $\nu \equiv \frac{\mu_{A}}{2 \pi}$, is irrational for if the linear orbits were periodic, nonlinear effects would start to play a more important role. One consequence of this choice is that the eigenvalues of $M_{A, A}$ are necessarily complex. $\beta_{A}$ causes the orbit to become an ellipse when it differs from 1 while $\alpha_{A}$ has the effect of skewing the ellipse.

\section{Number of Independent Orbit Data Sets}

As mentioned above, the BPMs can only measure the spatial coordinates of the beam and cannot measure its transverse momenta. Therefore for a 1Dimensional lattice a typical data set from an orbit may look like that in Table 2.1 below. At each turn the $x$ position of the beam is recorded as it passes by $B P M_{A}$, but the momentum $p_{x}$ is unknown.

The goal is to determine as much as we can about $M_{A, A}$ from this $x$-orbit data. The first question we might ask ourselves then is: How many independent $x$-orbit data sets can we use to determine $M_{A, A}$ ? The answer is that (after normalization) all orbits are actually the same, in that any given orbit will contain all other orbits. Therefore no new information can possibly be gained by taking more than $1 x$-orbit data set. The proof follows:

Consider two $x$-orbit data sets taken at $B P M_{A}: x_{0}, x_{1}, \ldots$ and $X_{0}, X_{1}, \ldots$ The first step in the proof that the second orbit, $X_{0}, X_{1}, \ldots$, is contained in the first is to discuss normalization:

\begin{tabular}{|c|ccccccc|}
\hline Turn $:$ & 0 & 1 & 2 & 3 & 4 & 5 & $\ldots$ \\
\hline$x_{A}$ & 3.1623 & 3.0075 & 2.5583 & 1.8587 & 0.9772 & 0.0000 & $\ldots$ \\
$p_{x A}$ & $p_{0}=?$ & $p_{1}=?$ & $p_{2}=?$ & $p_{3}=?$ & $p_{4}=?$ & $p_{5}=?$ & $\ldots$ \\
\hline
\end{tabular}

Table 1: Example $B P M_{A}$ data for a 1-Dimensional lattice 
Consider the evolution of the orbit $\overrightarrow{x_{0}}, \overrightarrow{x_{1}}, \ldots$ instead in normalized space. That is consider the orbit: $A_{A}^{-1} \overrightarrow{x_{0}}, A_{A}^{-1} \overrightarrow{x_{1}}, \ldots \equiv \overrightarrow{x_{0}^{N}}, \overrightarrow{x_{1}^{N}}, \ldots$ In normalized space the transfer matrix is simply $R_{A, A}$, a clockwise rotation. Thus:

$$
\begin{gathered}
\overrightarrow{x_{m+1}^{N}}=R_{A, A} \overrightarrow{x_{m}^{N}} \quad \rightarrow \\
\left|\overrightarrow{x_{m+1}^{N}}\right|=\left|\overrightarrow{x_{m}^{N}}\right| \equiv l \quad \forall m
\end{gathered}
$$

as $R_{A, A}$ conserves the norm. Similarly:

$$
\begin{gathered}
\overrightarrow{X_{m+1}^{N}}=R_{A, A} \overrightarrow{X_{m}^{N}} \quad \rightarrow \\
\left|\overrightarrow{X_{m+1}^{N}}\right|=\left|\overrightarrow{X_{m}^{N}}\right| \equiv L \quad \forall m
\end{gathered}
$$

Now:

$$
\overrightarrow{x_{m}}=\left(\begin{array}{cc}
\sqrt{\beta_{A}} & 0 \\
\frac{-\alpha_{A}}{\sqrt{\beta_{A}}} & \frac{1}{\sqrt{\beta_{A}}}
\end{array}\right) \overrightarrow{x_{m}^{N}}
$$

This implies that $x_{m}$ is maximized when $x_{m}^{N}$ is maximized. Now in normalized space $\overrightarrow{x_{m}^{N}}$ is rotating around a circle of radius $l$ in $x^{N}-p^{N}$ space. As $\nu$ is irrational $\rightarrow$ the normalized orbit $\overrightarrow{x_{0}^{N}}, \overrightarrow{x_{1}^{N}}, \ldots$ gets arbitrarily close to the $x^{N}$-axis, as the orbit of an irrational rotation is dense on the unit circle [2]. Therefore:

$$
\begin{gathered}
\max \left|x_{m}^{N}\right|=l \rightarrow \\
\max \left|x_{m}\right|=\sqrt{\beta_{A}} l
\end{gathered}
$$

Similarly:

$$
\begin{gathered}
\max \left|X_{m}^{N}\right|=L \rightarrow \\
\max \left|X_{m}\right|=\sqrt{\beta_{A}} L
\end{gathered}
$$

Therefore, measuring $\max \left|x_{m}\right|=\sqrt{\beta_{A}} l$ and $\max \left|X_{m}\right|=\sqrt{\beta_{A}} L$ and then dividing the two we obtain:

$$
\frac{\max \left|x_{m}\right|}{\max \left|X_{m}\right|}=l / L \equiv r
$$

We can now multiply the second orbit by $r$ to obtain a new orbit:

$$
\begin{gathered}
r \overrightarrow{X_{0}}, r \overrightarrow{X_{1}}, \ldots \equiv \overrightarrow{X_{0}^{\star}}, \overrightarrow{X_{1}^{\star}}, \ldots \\
r \overrightarrow{X_{0}^{N}}, r \overrightarrow{X_{1}^{N}}, \ldots \equiv \overrightarrow{X_{0}^{\star N}}, \overrightarrow{X_{1}^{\star N}}, \ldots
\end{gathered}
$$

We now have two orbits whose normalized phase space positions have the same norm:

$$
\left|\overrightarrow{x_{m}^{N}}\right|=\left|\overrightarrow{X_{n}^{\star N}}\right|=l \quad \forall m, n
$$


Once again, as $\nu$ is irrational $\rightarrow$ the orbit $\overrightarrow{x_{0}^{N}}, \overrightarrow{x_{1}^{N}}, \ldots$ is dense on the circle of radius $l$. As $\overrightarrow{X_{0}^{\star N}}$ lies on this circle $\rightarrow$ the orbit $\overrightarrow{x_{0}^{N}}, \overrightarrow{x_{1}^{N}}, \ldots$ must get arbitrarily close to $\overrightarrow{X_{0}^{\star N}}$. As it can get arbitrarily close, there must be some $j(k)$ such that:

$$
\left|\overrightarrow{x_{j(k)}^{N}}-\overrightarrow{X_{0}^{\star N}}\right|<10^{k}, \text { for any } \mathrm{k}
$$

Therefore we can choose a $k$ such that the difference between $\overrightarrow{x_{j(k)}^{N}}$ and $\overrightarrow{X_{0}^{\star N}}$ is unmeasurable. Doing this we find:

$$
\begin{gathered}
\overrightarrow{x_{j(k)}^{N}} \simeq \overrightarrow{X_{0}^{\star N}} \rightarrow R_{A, A} \overrightarrow{x_{j(k)}^{N}}=\overrightarrow{x_{j(k)+1}^{N}} \simeq R_{A, A} \overrightarrow{X_{0}^{\star N}}=\overrightarrow{X_{1}^{\star N}} \ldots \\
\rightarrow A_{A} \overrightarrow{x_{j(k)}^{N}}=\overrightarrow{x_{j(k)}} \simeq A_{A} \overrightarrow{X_{0}^{\star N}}=\overrightarrow{X_{0}^{\star}}, \overrightarrow{x_{j(k)+1}} \simeq \overrightarrow{X_{1}^{\star}} \ldots
\end{gathered}
$$

that is:

$$
\overrightarrow{x_{j(k)}}, \overrightarrow{x_{j(k)+1}}, \overrightarrow{x_{j(k)+2}}, \ldots \simeq \overrightarrow{X_{0}^{\star}}, \overrightarrow{X_{1}^{\star}}, \overrightarrow{X_{2}^{\star}}, \ldots
$$

where of course the two orbits will stay close for more turns the larger $k$ is chosen. This concludes the proof that any properly normalized second orbit will always be included (to an arbitrary degree of accuracy) within another arbitrarily chosen orbit. As a single orbit contains all other orbits, this implies we need only consider one when we are attempting to determine the parameters of our map.

\section{Specifying a Single $x$-Orbit}

We have seen above that you can get no new information about $M_{A, A}$ by using more than $1 x$-orbit data set. The next thing we will show is that in fact, only the first $3 x$ data points are required to specify an entire $x$-orbit. proof:

As $M_{A, A}$ is a real matrix with complex eigenvalues, the eigenvectors of $M_{A, A}$ must also be complex. As the zeroth turn of the orbit is a real vector, it cannot be an eigenvector of $M_{A, A}$. Therefore the first two turns of the orbit are independent and we can write, for some $a$ and $b$ :

$$
\left(\begin{array}{c}
x_{2} \\
p_{x 2}
\end{array}\right)=\left(\begin{array}{cc}
x_{0} & x_{1} \\
p_{x 0} & p_{x 1}
\end{array}\right)\left(\begin{array}{l}
a \\
b
\end{array}\right)
$$

That is, $\overrightarrow{x_{2}}$ is in the span of $\overrightarrow{x_{0}}$ and $\overrightarrow{x_{1}}$. Multiplying on the left by $M_{A, A}^{n}$ gives:

$$
\left(\begin{array}{c}
x_{n+2} \\
p_{x_{n+2}}
\end{array}\right)=\left(\begin{array}{cc}
x_{n} & x_{n+1} \\
p_{x_{n}} & p_{x_{n+1}}
\end{array}\right)\left(\begin{array}{c}
a \\
b
\end{array}\right)
$$

This gives 2 independent recursion relations for $x_{n}$ and $p_{x_{n}}$. By definition:

$$
\begin{gathered}
\left(\begin{array}{cc}
x_{n+1} & x_{n+2} \\
p_{x_{n+1}} & p_{x_{n+2}}
\end{array}\right)=M_{A, A}\left(\begin{array}{cc}
x_{n} & x_{n+1} \\
p_{x_{n}} & p_{x_{n+1}}
\end{array}\right) \rightarrow \\
\operatorname{det}\left(\begin{array}{cc}
x_{n+1} & x_{n+2} \\
p_{x_{n+1}} & p_{x_{n+2}}
\end{array}\right)=\operatorname{det}\left(\begin{array}{cc}
x_{n} & x_{n+1} \\
p_{x_{n}} & p_{x_{n+1}}
\end{array}\right) \equiv Q
\end{gathered}
$$


as $\operatorname{det} M_{A, A}=1$. Note that $Q \neq 0$ as the first two turns are linearly independent. From Eq.(27) we get the following two equations:

$$
\begin{gathered}
p_{x_{1}}=\frac{x_{1} p_{x_{0}}+Q}{x_{0}} \\
p_{x_{2}}=\frac{x_{2} p_{x_{1}}+Q}{x_{1}}=\frac{Q}{x_{1}}+\frac{x_{2} Q}{x_{1} x_{0}}+\frac{x_{2} p_{x_{0}}}{x_{0}}
\end{gathered}
$$

Plugging in Eq.(28) and Eq.(29) into Eq.(24) gives after a little manipulation:

$$
Q\left(\frac{x_{0}}{x_{1}}+\frac{x_{2}}{x_{1}}-b\right)=p_{x_{0}}\left(a x_{0}+b x_{1}-x_{2}\right)
$$

By Eq.(24) $a x_{0}+b x_{1}-x_{2}=0 \rightarrow$

$$
\frac{x_{0}}{x_{1}}+\frac{x_{2}}{x_{1}}-b=0 ;
$$

which finally gives:

$$
\begin{gathered}
b=\frac{x_{0}+x_{2}}{x_{1}} \\
a=-1
\end{gathered}
$$

Plugging in then to Eq.(25) we see that the entire $x$-orbit is specified uniquely by $x_{0}, x_{1}$, and $x_{2}$. Further the $x$-orbit depends only on these $3 x$ values and not at all on the $p_{x}$ values.

\section{Conclusion of Single BPM Analysis}

We are now ready to demonstrate that it is impossible to determine the matrix $M_{A, A}$ from the $x$-orbit data alone. In order to do this we will show how to construct an infinite set of matrices, all of which are symplectic and could produce the observed $x$-orbit data. As our only assumption on the form of $M_{A, A}$ is that it be symplectic we cannot determine which of the matrices in this set is the correct $M_{A, A}$ when using only the $x$-orbit data:

Let us begin by considering an observed $x$-orbit data set: $x_{0}, x_{1}, \ldots$ with the momenta unspecified as usual. Then we know:

$$
M_{A, A}=\left(\begin{array}{cc}
x_{1} & x_{2} \\
p_{x 1} & p_{x 2}
\end{array}\right)\left(\begin{array}{cc}
x_{0} & x_{1} \\
p_{x 0} & p_{x_{1}}
\end{array}\right)^{-1}
$$

$M_{A, A}$ is symplectic $\rightarrow$

$$
p_{x 2}=\frac{p_{x 1}\left(x_{0}+x_{2}\right)}{x_{1}}-p_{x 0}
$$

This reduces the number of unknown parameters specifying $M_{A, A}$ to 2 , those being $p_{x_{0}}$ and $p_{x_{1}}$. We are unable to measure these two momenta using the $B P M s$, but the question still remains if there is some way that you could find 
out what these are. We see that one cannot: For if you guess at $M_{A, A}$ by randomly choosing both a $p_{x g_{0}}$ and $p_{x g_{1}}$ and plug these into Eq.(34) you will obtain a different matrix:

$$
G_{A, A}\left(p_{x g_{0}}, p_{x g_{1}}\right)=\left(\begin{array}{cc}
x_{1} & x_{2} \\
p_{x g_{1}} & p_{x g_{2}}
\end{array}\right)\left(\begin{array}{cc}
x_{0} & x_{1} \\
p_{x g_{0}} & p_{x g_{1}}
\end{array}\right)^{-1}
$$

which is symplectic if $p_{x g_{2}}$ is obtained by plugging into Eq.(35). By definition, given input phase space position $\overrightarrow{x_{g 0}}=\left(x_{0}, p_{x g_{0}}\right)^{T}$ the orbit under $G_{A, A}\left(p_{x g_{0}}, p_{x g_{1}}\right)$ will have the same first three turn $x$ positions: $x_{0}, x_{1}, x_{2}$. As the entire $x_{n}$ orbit is determined by its first 3 values, this $x_{n}$ orbit will match that of the observed data set. Therefore as each of these matrices, $G_{A, A}\left(p_{x g_{0}}, p_{x g_{1}}\right)$, is symplectic and could produce the $x$-orbit observed we cannot determine which of them is the actual one turn map $M_{A, A}$.

We shall write $\mathcal{D}=2$, to signify the fact that there are 2 degrees of freedom in $M_{A, A}$ left unspecified by the observable data alone. Thus 2 extra constraints in addition to the $x$-orbit data from a single $B P M$ are required to determine the 1-turn map $M_{A, A}$ in a 1-dimensional lattice.

\subsection{Multiple BPM Analysis}

Now that we have proven that it is impossible to determine $M_{A, A}$ given just the $x$-orbit data from $B P M_{A}$ the next question we must answer is whether or not it may be possible to determine $M_{A, A}$ using the $x$-orbit data from multiple $B P M$ s around the ring in conjunction. The answer, as we shall see, is that again you cannot.

We begin by looking at a hypothetical storage ring which has $2 B P M \mathrm{~s}$. As the beam circles around the ring the $x$-position of the beam is recorded turn by turn as it passes by $B P M_{A}$ and then again as it passes by $B P M_{B}$. We define an orbit data set as a complete set of data for all $B P M \mathrm{~s}$ under consideration for a given run. Of course the transverse momenta is still unmeasurable at each of the BPMs and so a typical orbit data set may look like that in Table 2.2 below.

As in the single $B P M$ proof above, our analysis of the double $B P M$ case will hinge on the construction of an infinite set of symplectic matrices, $G_{A, A}(a, b, \ldots)$ and $G_{B, A}(a, b, \ldots)$, which are capable of producing the $x_{n}$-orbits observed in the orbit data sets. First, however, we must turn our attention to the number of independent orbit data sets we may use.

\begin{tabular}{|c|ccccccc|}
\hline Turn $:$ & 0 & 1 & 2 & 3 & 4 & 5 & $\ldots$ \\
\hline$x_{A}$ & 3.0046 & 3.0391 & 2.5351 & 1.5817 & 0.3480 & -0.9473 & $\ldots$ \\
$p_{x}$ & $p_{0}=?$ & $p_{1}=?$ & $p_{2}=?$ & $p_{3}=?$ & $p_{4}=?$ & $p_{5}=?$ & $\ldots$ \\
$x_{B}$ & 3.4580 & 3.4345 & 2.8022 & 1.6734 & 0.2479 & -1.2215 & $\ldots$ \\
$p_{x B}$ & $p_{0}=?$ & $p_{1}=?$ & $p_{2}=?$ & $p_{3}=?$ & $p_{4}=?$ & $p_{5}=?$ & $\ldots$ \\
\hline
\end{tabular}

Table 2: Example $B P M_{A}$ and $B P M_{B}$ data for a 1-Dimensional lattice 


\section{Number of Independent Orbit Data Sets}

As in the analogous section above, we wish to show that all orbit data sets are contained (to any desired degree of accuracy) within every other orbit data set. We can easily extend the result we obtained above:

Consider two orbit data sets, each consisting of the orbit data from $B P M_{A}$ and the data from $B P M_{B}$ :

$$
\operatorname{set} 1:\left(\overrightarrow{x_{0, A}}, \overrightarrow{x_{1, A}}, \ldots ; \overrightarrow{x_{0, B}}, \overrightarrow{x_{1, B}}, \ldots\right) \quad \operatorname{set} 2:\left(\overrightarrow{X_{0, A}}, \overrightarrow{X_{1, A}}, \ldots ; \overrightarrow{X_{0, B}}, \overrightarrow{X_{1, B}}, \ldots\right)
$$

If we now plug in the orbits $x_{n, A}$ and $X_{n, A}$ into Eq.(16) we shall obtain the normalization ratio $r_{A}$. Multiplying both the orbit $\left(\overrightarrow{X_{n, A} \ldots}\right)$ and the orbit $\left(\overrightarrow{X_{m, B} \ldots}\right)$ by $r_{A}$, we obtain the normalized orbit data set, $\left(\overrightarrow{X_{n, A}^{\star} \ldots}\right)$ and $\left(\overrightarrow{X_{m, B}^{\star} \ldots}\right)$. Now by Eq.(20) we can find a $j(k)$ such that the following holds:

$$
\left|\overrightarrow{x_{j(k), A}^{N}}-\overrightarrow{X_{0, A}^{\star N}}\right|<10^{k}, \text { for any } \mathrm{k}
$$

If we choose a large $k$ then, we have by Eq.(23) that:

$$
\overrightarrow{x_{j(k), A}}, \overrightarrow{x_{j(k)+1, A}}, \overrightarrow{x_{j(k)+2, A}}, \ldots \simeq \overrightarrow{X_{0, A}^{\star}}, \overrightarrow{X_{1, A}^{\star}}, \overrightarrow{X_{2, A}^{\star}}, \ldots
$$

where the two orbits stay close for more turns the larger $\mathrm{k}$ is chosen. Multiplying on the left by $M_{B, A}$ we obtain:

$$
\begin{array}{r}
M_{B, A} \overrightarrow{x_{j(k), A}}, M_{B, A} \overrightarrow{x_{j(k)+1, A}}, \ldots=\overrightarrow{x_{j(k), B}}, \overrightarrow{x_{j(k)+1, B}}, \ldots \\
\simeq M_{B, A} \overrightarrow{X_{0, A}^{\star}}, M_{B, A} \overrightarrow{X_{1, A}^{\star}}, \ldots=\overrightarrow{X_{0, B}^{\star}}, \overrightarrow{X_{1, B}^{\star}}, \ldots \rightarrow \\
\stackrel{x_{j(k), B}}{\longrightarrow} \overrightarrow{x_{j(k)+1, B}}, \overrightarrow{x_{j(k)+2, B}}, \ldots \simeq \overrightarrow{X_{0, B}^{\star}}, \overrightarrow{X_{1, B}^{\star}}, \overrightarrow{X_{2, B}^{\star}}, \ldots
\end{array}
$$

showing that the 2 nd orbit at $B$ is contained in the first as well. Clearly this argument can be extended to any number of $B P M$ s by proceeding inductively. In general we see that for 1-dimensional lattices a single orbit data set contains all others, implying we need only consider one when attempting to determine the matrices $M_{A, A}, M_{B, A}, M_{C, B}$, etc..

\section{Construction of Guess Matrices}

By definition, the actual matrix $M_{B, A}$ satisfies the following relation:

$$
M_{B, A}\left(\begin{array}{cc}
x_{0, A} & x_{1, A} \\
p_{x_{0, A}} & p_{x_{1, A}}
\end{array}\right)=\left(\begin{array}{cc}
x_{0, B} & x_{1, B} \\
p_{x_{0, B}} & p_{x_{1, B}}
\end{array}\right)
$$

from which we obtain,

$$
M_{B, A}=\left(\begin{array}{cc}
x_{0, B} & x_{1, B} \\
p_{x_{0, B}} & p_{x_{1, B}}
\end{array}\right)\left(\begin{array}{cc}
x_{0, A} & x_{1, A} \\
p_{x_{0, A}} & p_{x_{1, A}}
\end{array}\right)^{-1}
$$


$M_{A, A}$ is given by Eq.(34). Again the symplectic condition Eq.(35) still holds for $M_{A, A}$, while setting the determinant of Eq.(41) to 1 we see that the symplectic condition applied to $M_{B, A}$ is equivalent to:

$$
p_{x_{1, B}}=\frac{x_{1, B}}{x_{0, B}} p_{x_{0, B}}-\frac{x_{1, A}}{x_{0, B}} p_{x_{0, A}}+\frac{x_{0, A}}{x_{0, B}} p_{x_{1, A}}
$$

Thus the symplectic condition reduces the number of unknown parameters to 3 : $p_{x_{0, A},}, p_{x_{1, A}}$, and $p_{x_{0, B}}$. Choosing random values for these momenta we can con-

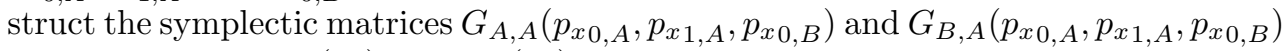
by plugging into Eq.(34) and Eq.(41):

$$
\begin{aligned}
G_{A, A} & =\left(\begin{array}{cc}
x_{1, A} & x_{2, A} \\
p_{x g_{1, A}} & p_{x g_{2, A}}
\end{array}\right)\left(\begin{array}{cc}
x_{0, A} & x_{1, A} \\
p_{x g_{0, A}} & p_{x g_{1, A}}
\end{array}\right)^{-1} \\
G_{B, A} & =\left(\begin{array}{cc}
x_{0, B} & x_{1, B} \\
p_{x g_{0, B}} & p_{x g_{1, B}}
\end{array}\right)\left(\begin{array}{cc}
x_{0, A} & x_{1, A} \\
p_{x g_{0, A}} & p_{x g_{1, A}}
\end{array}\right)^{-1}
\end{aligned}
$$

By the above, given input data $\overrightarrow{x_{g 0, A}}=\left(x_{0, A}, p_{x g_{0, A}}\right)^{T}$ we know that the $x_{n, A}$ orbit under $G_{A, A}$ will match that of the observed orbit data set. By definition the $x_{g 0, B}$, and $x_{g 1, B}$ values of the first two turns will also match the authentic data set, where:

$$
\left(\begin{array}{c}
x_{g n, B} \\
p_{x g_{n, B}}
\end{array}\right)=G_{B, A}\left(\begin{array}{c}
x_{n, A} \\
p_{x g_{n, A}}
\end{array}\right)
$$

If we could show that $x_{g 2, B}$ also matches the observed orbit data set, then the first $3 x_{g n, B}$ values would match. This would then imply that the entire $x_{g n, B}$ orbit matches the observed orbit data. Apply $G_{B, A}$ to obtain the third x-value in the orbit:

$$
\left(\begin{array}{c}
x_{g 2, B} \\
p_{x g_{n, B}}
\end{array}\right)=\left(\begin{array}{cc}
x_{0, B} & x_{1, B} \\
p_{x g_{0, B}} & p_{x g_{1, B}}
\end{array}\right)\left(\begin{array}{cc}
x_{0, A} & x_{1, A} \\
p_{x g_{0, A}} & p_{x g_{1, A}}
\end{array}\right)^{-1}\left(\begin{array}{c}
x_{2, A} \\
p_{x g_{2, A}}
\end{array}\right)
$$

By Eq.(24):

$$
\left(\begin{array}{cc}
x_{0, A} & x_{1, A} \\
p_{x g_{0, A}} & p_{x g_{1, A}}
\end{array}\right)^{-1}\left(\begin{array}{c}
x_{2, A} \\
p_{x g_{2, A}}
\end{array}\right)=\left(\begin{array}{c}
a \\
b
\end{array}\right)
$$

Using Eq.(32), and Eq.(33) and plugging into Eq.(46) we get,

$$
\left(\begin{array}{c}
x_{g 2, B} \\
p_{x g_{n, B}}
\end{array}\right)=\left(\begin{array}{cc}
x_{0, B} & x_{1, B} \\
p_{x g_{0, B}} & p_{x g_{1, B}}
\end{array}\right)\left(\begin{array}{c}
-1 \\
\frac{x_{0, A}+x_{2, A}}{x_{1, A}}
\end{array}\right)
$$

Explicitly showing that $x_{g 2, B}$ depends only on $x_{0, B}, x_{1, B}, x_{0, A}, x_{1, A}$, and $x_{2, A}$ but is completely independent of all $A$ and $B$ momenta. As all of these $x$ values match the authentic data, it follows that $x_{g 2, B}=x_{2, B}$ and therefore $x_{g n, B}=$ $x_{n, B} \forall n$.

Thus we have shown that the matrices $G_{A, A}\left(p_{x_{0}, A}, p_{x_{1, A}}, p_{x_{0, B}}\right)$, and $G_{B, A}\left(p_{x_{0, A}}, p_{x_{1, A}}, p_{x_{0, B}}\right)$ given in Eq.(43) and Eq.(44) are symplectic and could 
produce the observed $x_{n}$-orbits at both $B P M_{A}$ and $B P M_{B}$ for any choice of the values $p_{x_{0, A}}, p_{x_{1, A}}$, and $p_{x_{0, B},}$. Therefore the observable $x$-orbit data from $2 B P M \mathrm{~s}$ is insufficient to determine the matrices $M_{A, A}$ and $M_{B, A}$ and leaves the global number of undetermined parameters in these matrices at $\mathcal{D}=3$.

\section{Extension to Multiple BPMs}

We may extend this result to multiple $B P M$ s by proceeding inductively. Take, for example, a storage ring consisting of $3 B P M \mathrm{~s}$. By the above, we need only consider a single orbit data set in the following.

Looking first at only $B P M_{A}$ and $B P M_{B}$ construct the matrices $G_{A, A}$ and $G_{B, A}$ using Eq.(43) and Eq.(44). Now construct $G_{C, B}$ by randomly choosing $p_{x g_{0, C}}$ :

$$
G_{C, B}=\left(\begin{array}{cc}
x_{0, C} & x_{1, C} \\
p_{x g_{0, C}} & p_{x g_{1, C}}
\end{array}\right)\left(\begin{array}{cc}
x_{0, B} & x_{1, B} \\
p_{x g_{0, B}} & p_{x g_{1, B}}
\end{array}\right)^{-1}
$$

Where $p_{x g_{1, B}}$ is given by Eq.(42) and the symplectic condition on $G_{C, B}$ gives $p_{x g_{1, C}}$ :

$$
p_{x g 1, C}=\frac{x_{1, C}}{x_{0, C}} p_{x g 0, C}-\frac{x_{1, B}}{x_{0, C}} p_{x g 0, B}+\frac{x_{0, B}}{x_{0, C}} p_{x g 1, B}
$$

The symplectic condition on $G_{A, A}, G_{B, A}$ and $G_{C, B}$ reduces the number of unknown parameters to 4 . Similar to the above we can see that no matter what our choice is for these 4 parameters, we will generate a consistent set of symplectic matrices which could generate orbits with $x_{n}$-orbits matching that of the observed orbit data set. As the $x$ data from the orbit data set is all the information we know, we cannot possibly reduce the number unknown parameters specifying the system to less than 4 .

Continuing in this fashion we obtain the following result: The constraints supplied by the observable data from $N B P M$ s alone are insufficient to determine the symplectic matrices $M_{A, A}, M_{B, A}, \ldots, M_{N, N-1}$ and leaves the global number of unknown parameters specifying these matrices at $\mathcal{D}=\mathrm{N}+1$.

\subsection{Results in Terms of Standard Parameters}

In this section we will quickly describe the work above in terms of the machine parameters we are interested in determining, namely the $\alpha$ 's, $\beta$ 's, and $\mu$ 's.

The only parameters that can be determined from the observable data alone are the $\mu$ 's. We can see intuitively that $\mu_{A}=\mu_{B}=\mu_{c}=\ldots \equiv \mu$ and this can be proven mathematically without too much difficulty. To find $\mu$ from the $x$ data one need only set up the recursion relation Eq.(25) and solve for the roots of its characteristic equation. These roots will have the form $e^{ \pm \imath \mu}$.

From Eq.(13), we see that $\max \left|x_{m, A}\right|=\sqrt{\beta_{A}} l$ and $\max \left|x_{m, B}\right|=\sqrt{\beta_{B}} l$. From these two we may write: $\beta_{B}=\left(\frac{\max \left|x_{m, B}\right|}{\max \left|x_{m, A}\right|}\right)^{2} \beta_{A}$. Similarly, we may write $\beta_{C}$ in terms of $\beta_{A}$ and so on. Therefore once $\beta_{A}$ is specified, all other $\beta_{\mathrm{s}}$ can be determined from the orbit data. 
If there are $N B P M s$ in our storage ring, then by the above, the measurable orbit data leaves $N+1$ parameters unspecified. Note that in terms of the $\alpha_{A}, \alpha_{B}, \alpha_{C} \ldots, \beta_{A}, \beta_{B}, \beta_{C} \ldots$, and $\mu$ parameters, we have already reduced the number required to specify them all to $N+1$, those being the $N \alpha \mathrm{s}$, (one at each $B P M$ ) and $\beta_{A}$. As these parameters completely specify our system, this must mean that the $N \alpha$ s and $\beta_{A}$ are unspecified by the data and are independent: even if you somehow knew $N$ of the parameters $\alpha_{A}, \alpha_{B}, \ldots, \alpha_{N}$, and $\beta_{A}$, you still could not determine the $N+1^{\text {st }}$ from the observable data alone.

Note: To provide some physical intuition as to why we cannot determine all the parameters $\alpha_{A}, \alpha_{B}$, etc., consider a lattice with just a single $B P M$. We can measure only the $x$-orbit values and not the momenta. But the values $x_{n}$ are simply given by, $x_{n}=\sqrt{\beta_{A}} x_{n}^{N}$, which is completely independent on $\alpha_{A}$ and so we cannot hope (at least in the single $B P M$ case) to get at $\alpha_{A}$ from this data.

\subsection{Approximate Solution}

As we cannot determine the matrices $M_{A, A}, M_{B, A}$, etc. from the measurable data alone, we must resort to approximate solution techniques. A reasonable approximation can be obtained for 2 or more $B P M \mathrm{~s}$ when the $B P M \mathrm{~s}$ are spaced close to one another around the ring. We may approximate the transverse motion of the particle beam between the quadrupole magnets as being essentially a drift:

$$
D=\left(\begin{array}{ll}
1 & \delta \\
0 & 1
\end{array}\right)
$$

where $\delta$ is determined by measuring the distance between the two magnets. Meanwhile, as the beam passes by one of the quadrupole magnets it is either focused or defocused:

$$
\begin{aligned}
& F_{x}=\left(\begin{array}{cc}
1 & 0 \\
-k & 1
\end{array}\right) \\
& F_{y}=\left(\begin{array}{ll}
1 & 0 \\
k & 1
\end{array}\right)
\end{aligned}
$$

$F_{x}$ represent an $x$-focusing matrix, $F_{y}$ represents an $x$-defocusing matrix. If we approximate the matrix $M_{B, A}$ as a product of these 3 types of matrices we can then easily determine $M_{A, A}$. Assuming $M_{B, A}$ known:

$$
\begin{aligned}
M_{B, A} & =\left(\begin{array}{cc}
a & b \\
c & d
\end{array}\right) \rightarrow \\
\left(\begin{array}{cc}
a & b \\
c & d
\end{array}\right)\left(\begin{array}{c}
x_{n, A} \\
p_{x_{n, A}}
\end{array}\right) & =\left(\begin{array}{c}
x_{n, B} \\
p_{x_{n}, B}
\end{array}\right) \rightarrow \\
p_{x_{n, A}} & =\frac{1}{b} x_{n, B}-\frac{a}{b} x_{n, A}
\end{aligned}
$$


Plugging these momenta values into Eq.(34) we obtain $M_{A, A}$ and the entire set of parameters is determined.

\subsection{A Note on Rational Tunes}

The results above assumed that the tune $\nu=\frac{\mu}{2 \pi}$ is irrational. As I mentioned before, this is the case in PEPII as a rational tune would result in nonlinear resonance effects. However, if one chose a rational tune which would result in very large periods, e.g. $\nu \equiv \frac{1}{10^{50}}(\bmod 1)$, then the resonance effects would be minimal. If $\nu$ were chosen so, would it then be possible to determine the map $M_{A, A}$ ? We prove below that it is not:

Assume that for some 1-turn map $M_{A, A}$ with rational tune $\nu$ you could determine the matrix $M_{A, A}$ using only the observable data. The set of irrationals is dense in the set of reals and therefore we may find an irrational $\nu_{2}$ infinitely close to $\nu$. Consider now a second 1-turn map $M_{A, A, 2}$ which is identical to $M_{A, A}$ except that it's tune is $\nu_{2}$ rather than $\nu$. The orbit data you would measure under $M_{A, A, 2}$ would be infinitely close to that from $M_{A, A}$ and therefore you would be able to use the same technique used to determine $M_{A, A}$ to get an approximation to $M_{A, A, 2}$ which is infinitely accurate, if not exact. However, we have shown above that it is not possible to accurately determine the matrix $M_{A, A, 2}$ and therefore our original assumption must be wrong: it is not possible to determine the matrix $M_{A, A}$ for any rational tune $\nu$. (further it's not possible to accurately determine anything with a rational $\nu$ that you couldn't with an irrational $\nu$.) This result can easily be extended to lattices with multiple $B P M \mathrm{~s}$ and applies to 2-dimensional lattices as well.

\section{Two-Dimensional Case}

We can now apply what we have learned from the 1-Dimensional lattice problem above to the more relevant 2-Dimensional problem which describes the lattice in PEPII. In section 3.1 we shall look at what can be determined from data from a single $B P M$ alone, in section 3.2 we will analyze lattices containing 2 or more $B P M^{\prime} s$, and in section 3.3 we will discuss how many $B P M \mathrm{~s}$ are required to determine the machine parameters when the matrices $M_{B, A}, M_{C, B}$, etc. are assumed known.

\subsection{Single BPM Analysis}

In this section we shall prove that given $x$ and $y$ orbit data from a single $B P M$ it is impossible to determine the 1-turn map $M_{A, A}$ and that there are 8 degrees of freedom left unspecified by the data alone. 


\section{Symplectic Matrices Revisited}

A $4 \times 4$ symplectic matrix $M$ is defined as one such that:

$$
M^{T} S_{4 x 4} M=S_{4 x 4}
$$

where,

$$
S_{4 x 4} \equiv\left(\begin{array}{cc}
S_{2 x 2} & 0_{2 x 2} \\
0_{2 x 2} & S_{2 x 2}
\end{array}\right)
$$

It can be shown [3] that in the 2-dimensional case the symplectic matrices $M_{N, M}$ can be decomposed in a form analogous to that in Eq.(5):

$$
\begin{aligned}
M_{N, M} & =\widetilde{A}_{N} R_{N, M} \widetilde{A}_{M}^{-1} \\
& =C_{N} A_{N} R_{N, M} A_{M}^{-1} C_{M}^{-1}
\end{aligned}
$$

where $R_{N, M}, A_{M}$, and $C_{M}$ have the following forms:

$$
\begin{aligned}
R_{N, M} & =\left(\begin{array}{cc}
R_{x N, M} & 0_{2 x 2} \\
0_{2 x 2} & R_{y_{N, M}}
\end{array}\right) \\
A_{M} & =\left(\begin{array}{cc}
A_{x, M} & 0_{2 x 2} \\
0_{2 x 2} & A_{y, M}
\end{array}\right) \\
C_{M} & =\left(\begin{array}{cc}
I_{2 x 2} \cos \phi & -S_{2 x 2} W^{T} S_{2 x 2} \sin \phi \\
-W \sin \phi & I_{2 x 2} \cos \phi
\end{array}\right)
\end{aligned}
$$

$R_{x N, M}, R_{y_{N, M}}, A_{x M}$, and $A_{y_{M}}$ have the same form as those in Eq.(6) and $W$ is a $2 x 2$ matrix:

$$
W=\left(\begin{array}{ll}
a & b \\
c & d
\end{array}\right)
$$

If we assume that all elements of $W$ are nonzero, it can be shown that we can choose $d \equiv d(a, b, c)$ such that $C$ is symplectic. Thus there are 10 unknown parameters specifying the symplectic matrix $M_{A, A}$ :

$$
\alpha_{x}, \beta_{x}, \alpha_{y}, \beta_{y}, \mu_{x}, \mu_{y}, \phi, a, b \text {, and } c
$$

In addition to these 10, there are 4 unknown parameters describing errors in how the $B P M$ measures the position of the beam. These are $g_{x}, g_{y}, \theta_{x, y}$, and $\theta_{y, x}$ and they are defined as follows: if the actual transverse position of the beam is $(x, y)$ then the $B P M$ will read out:

$$
\begin{aligned}
& x_{R}=g_{x} x+\theta_{x y} y \\
& y_{R}=g_{y} y+\theta_{y x} x
\end{aligned}
$$

This brings the number of unknown parameters specifying the measurement error and the one turn map at $B P M_{A}$ to 14 .

Looking back at the decomposition Eq.(57), we see that $C_{M}^{-1}$ transforms into an uncoupled space, consisting of two $2 x 2$ subspaces, the $x^{U}-p_{x}^{U}$ and $y^{U}-p_{y}^{U}$ 
eigenplanes (the $U$ superscript denoting the uncoupled space). Thus in this space the orbits may be decomposed as the conjunction of two independent 1-Dimensional lattice orbits, one in each eigenplane. Again the matrix $A_{M}^{-1}$ transforms into the normalized space in which the orbits in each eigenplane are circular.

\section{Number of Independent Orbits}

In this section we shall demonstrate that it is possible to manipulate an orbit data set by normalizing its two independent 1-dimensional orbits in the normalized space so that the new normalized orbit data set will be contained in a second orbit data set. For simplicity, we shall ignore the measurement errors associated with the parameters $g_{x}, g_{y}, \theta_{x, y}$, and $\theta_{y, x}$ in this section. However, it can easily be seen that the same method described below is still applicable when these parameters are included. We begin by describing the normalization process:

In the normalized space we have,

$$
\left(\begin{array}{c}
x_{n}^{N} \\
p_{x_{n}}^{N}
\end{array}\right)=\left(\begin{array}{cc}
\cos n \mu_{x} & \sin n \mu_{x} \\
-\sin n \mu_{x} & \cos n \mu_{x}
\end{array}\right)\left(\begin{array}{c}
x_{0}^{N} \\
p_{x 0}
\end{array}\right)
$$

Multiplying on the left by $A_{x}$ we must have,

$$
\left(\begin{array}{c}
x_{n}^{U} \\
p_{x_{n}}^{U}
\end{array}\right)=\left(\begin{array}{cc}
s & t \\
u & v
\end{array}\right)\left(\begin{array}{c}
\cos n \mu_{x} \\
\sin n \mu_{x}
\end{array}\right)
$$

for some constants $s, t, u$, and $v$. Similarly we have,

$$
\left(\begin{array}{c}
y_{n}^{U} \\
p_{y_{n}}^{U}
\end{array}\right)=\left(\begin{array}{cc}
w & x \\
y & z
\end{array}\right)\left(\begin{array}{c}
\cos n \mu_{y} \\
\sin n \mu_{y}
\end{array}\right)
$$

for some constants $w, x, y$, and $z$. Multiplying $\overrightarrow{x_{n}^{U}}=\left(x_{n}^{U}, p_{x_{n}}^{U}, y_{n}^{U}, p_{y_{n}}^{U}\right)^{T}$ on the left by $C_{A}$ we obtain:

$$
\begin{aligned}
& x_{n}=j \cos n \mu_{x}+k \sin n \mu_{x}+l \cos n \mu_{y}+m \sin n \mu_{y} \\
& y_{n}=n \cos n \mu_{x}+o \sin n \mu_{x}+p \cos n \mu_{y}+q \sin n \mu_{y}
\end{aligned}
$$

$j, k, l, m, n, o, p$, and $q$ all constants. To determine these coefficients we must first find the values of $\mu_{x}$ and $\mu_{y}$. These can be determined by setting up a recursion relation like that in Eq.(25) and then solving for the roots of its characteristic equation. Once the values of $\mu_{x}$ and $\mu_{y}$ are known we can determine the coefficients $j, k, l, m, n, o, p$, and $q$ using the first 4 positions of the orbit. Noting the form of $C_{A}$ and $A_{A}$, we must have:

$$
\begin{aligned}
& j \cos n \mu_{x}+k \sin n \mu_{x}=\cos \phi \sqrt{\beta_{x}} x_{n}^{N} \\
& p \cos n \mu_{y}+q \sin n \mu_{y}=\cos \phi \sqrt{\beta_{y}} y_{n}^{N}
\end{aligned}
$$


Writing, $\left|\left(x_{n}^{N}, p_{x}^{N}\right)^{T}\right| \equiv l_{x}$ and $\left|\left(y_{n}^{N}, p_{y_{n}}^{N}\right)^{T}\right| \equiv l_{y}$ (which are independent of $n$ as the normalized orbit in each eigenplane lies on a circle) and recalling that $\mu_{x}$ and $\mu_{y}$ are irrational we must have:

$$
\max \left(j \cos n \mu_{x}+k \sin n \mu_{x}\right)=|\cos \phi| \sqrt{\beta_{x}} l_{x}
$$

and,

$$
\max \left(p \cos n \mu_{y}+q \sin n \mu_{y}\right)=|\cos \phi| \sqrt{\beta_{y}} l_{y}
$$

Considering now a second orbit data set $\overrightarrow{X_{n}}=\left(X_{n}, P_{x_{n}}, Y_{n}, P_{y_{n}}\right)^{T}$, we have:

$$
\begin{gathered}
X_{n}=J \cos n \mu_{x}+K \sin n \mu_{x}+L \cos n \mu_{y}+M \sin n \mu_{y} \\
Y_{n}=N \cos n \mu_{x}+O \sin n \mu_{x}+P \cos n \mu_{y}+Q \sin n \mu_{y}
\end{gathered}
$$

with,

$$
\begin{aligned}
& \max \left(J \cos n \mu_{x}+K \sin n \mu_{x}\right)=\cos \phi \sqrt{\beta_{x}} L_{x} \\
& \max \left(P \cos n \mu_{y}+Q \sin n \mu_{y}\right)=\cos \phi \sqrt{\beta_{y}} L_{y}
\end{aligned}
$$

Dividing Eq.(70) by Eq.(74) and Eq.(71) by Eq.(75) we obtain,

$$
\begin{aligned}
& \frac{\max \left(j \cos n \mu_{x}+k \sin n \mu_{x}\right)}{\max \left(J \cos n \mu_{x}+K \sin n \mu_{x}\right)}=\frac{l_{x}}{L_{x}} \equiv r_{x} \\
& \frac{\max \left(p \cos n \mu_{y}+q \sin n \mu_{y}\right)}{\max \left(P \cos n \mu_{y}+Q \sin n \mu_{y}\right)}=\frac{l_{y}}{L_{y}} \equiv r_{y}
\end{aligned}
$$

If we now multiply all terms of the orbit $\overrightarrow{X_{n}}$ containing $\mu_{x}$ by $r_{x}$ and all terms containing $\mu_{y}$ by $r_{y}$, we obtain a new orbit $\overrightarrow{X_{n}^{\star}}$. For example the $X_{n}^{\star}$ orbit will be given by:

$$
X_{n}^{\star}=r_{x} J \cos n \mu_{x}+r_{x} K \sin n \mu_{x}+r_{y} L \cos n \mu_{y}+r_{y} M \sin n \mu_{y}
$$

In effect, we have constructed another observable orbit, $\overrightarrow{X_{n}^{\star}}$, s.t. $\left|\left(X_{n}^{\star N}, P_{x n}^{\star N}\right)^{T}\right|=$ $l_{x}$ and $\left|\left(Y_{n}^{\star N}, P_{y_{n}}^{\star N}\right)^{T}\right|=l_{y}$. Thus in normalized space, $\left(X_{n}^{\star N}, P_{x_{n}}^{\star N}\right)^{T}$ is rotating around in a circle which has the same radius as the circular orbit $\left(x_{n}^{N}, p_{x n}^{N}\right)^{T}$ and $\left(Y_{n}^{\star N}, P_{y_{n}}^{\star N}\right)^{T}$ is rotating around in a circle which has the same radius as the circular orbit $\left(y_{n}^{N}, p_{y_{n}}^{N}\right)^{T}$.

The rotation angles $\mu_{x}$ and $\mu_{y}$ are always chosen not equal as this results in resonances. As they are both irrational, it can then be shown that there must be some $j(k)$ such that:

$$
\left|\left(X_{0}^{\star N}, P_{x 0}^{\star N}\right)^{T}-\left(x_{j(k)}^{N}, p_{x j(k)}^{N}\right)^{T}\right|<10^{k}
$$

and,

$$
\left|\left(Y_{0}^{\star N}, P_{y_{0}}^{\star N}\right)^{T}-\left(y_{j(k)}^{N}, p_{y_{j(k)}}^{N}\right)^{T}\right|<10^{k} \text {, for any } \mathrm{k}
$$

Multiplying on the left by $C_{A} A_{A}$ we find,

$$
\overrightarrow{x_{j(k)}}, \overrightarrow{x_{j(k)+1}}, \overrightarrow{x_{j(k)+2}}, \ldots \simeq \overrightarrow{X_{0}^{\star}}, \overrightarrow{X_{1}^{\star}}, \overrightarrow{X_{2}^{\star}}, \ldots
$$


where the two orbits will stay close for more turns the larger $k$ is chosen. Thus the second normalized orbit is contained in the first.

Although the "normalization" process described here is not as simple as the scaling factor used in the 1-dimensional process, they amount to the same thing: we can take a single orbit data set and from it construct all others. This implies no new information about the map can be obtained by taking more data and again we need only consider one orbit data set when attempting to determine $M_{A, A}$.

\section{Construction of Guess Matrices}

Consider a single orbit data set $\overrightarrow{x_{0, R}}, \overrightarrow{x_{1, R}}, \ldots$ We shall construct a set of symplectic guess matrices $G_{B, A}$ which produce the observed $x_{0, R}, x_{1, R}, \ldots$ and $y_{0, R}, y_{1, R}, \ldots$ orbits. Begin by randomly choosing $g_{x}, g_{y}, \theta_{x y}$, and $\theta_{y x}$. Inverting Eq.(62) we obtain:

$$
\left(\begin{array}{l}
x_{n, g} \\
y_{n, g}
\end{array}\right)=\frac{1}{g_{x, g} g_{y, g}-\theta_{x y, g} \theta_{y x, g}}\left(\begin{array}{cc}
g_{y, g} & -\theta_{x y, g} \\
-\theta_{y x, g} & g_{x, g}
\end{array}\right)\left(\begin{array}{l}
x_{n, R} \\
y_{n, R}
\end{array}\right)
$$

obtaining a guess at the actual position of the beam for each turn of the orbit. Now randomly choose the parameters determining the matrix $C_{A}$. These are: $a, b, c$, and $\phi($ recall $d \equiv d(a, b, c))$.

$$
C_{A, g}=\left(\begin{array}{cc}
I_{2 x 2} \cos \phi_{g} & -S_{2 x 2} W_{g}^{T} S_{2 x 2} \sin \phi_{g} \\
-W_{g} \sin \phi_{g} & I_{2 x 2} \cos \phi_{g}
\end{array}\right)
$$

Similar to Eq.(66) and Eq.(67) we may decompose $x_{n, g}$ and $y_{n, g}$ as follows:

$$
\begin{aligned}
& x_{n, g}=j \cos n \mu_{x}+k \sin n \mu_{x}+l \cos n \mu_{y}+m \sin n \mu_{y} \\
& y_{n, g}=n \cos n \mu_{x}+o \sin n \mu_{x}+p \cos n \mu_{y}+q \sin n \mu_{y}
\end{aligned}
$$

for some new constants $j, k, l, m, n, o, p$, and $q$ which can again be solved for by using the first 4 positions of the orbit. From Eq.(57) and Eq.(60) we obtain,

$$
\begin{aligned}
& x_{n, g}=\cos \phi_{g} x_{n, g}^{U}+d_{g} \sin \phi_{g} y_{n, g}^{U}-b_{g} \sin \phi_{g} p_{y_{n, g}}^{U} \\
& y_{n, g}=\cos \phi_{g} y_{n, g}^{U}-a_{g} \sin \phi_{g} x_{n, g}^{U}-b_{g} \sin \phi_{g} p_{x_{n, g}}^{U}
\end{aligned}
$$

comparison with Eq.(84) and Eq.(85) gives:

$$
\begin{aligned}
& x_{n, g}^{U}=\frac{1}{\cos \phi_{g}}\left(j \cos n \mu_{x}+k \sin n \mu_{x}\right) \\
& y_{n, g}^{U}=\frac{1}{\cos \phi_{g}}\left(p \cos n \mu_{y}+q \sin n \mu_{y}\right)
\end{aligned}
$$

Plugging these back into Eq.(86) and Eq.(87),

$$
\begin{aligned}
p_{x, y}^{U} & =\frac{-1}{b_{g} \sin \phi_{g}}\left[n \cos n \mu_{x}+o \sin n \mu_{x}+a_{g} \frac{\sin \phi_{g}}{\cos \phi_{g}}\left(j \cos n \mu_{x}+k \sin n \mu_{x}\right)\right] \\
& =-\left[\frac{a_{g} j}{b_{g} \cos \phi_{g}}+\frac{n}{b_{g} \sin \phi_{g}}\right] \cos n \mu_{x}-\left[\frac{a_{g} k}{b_{g} \cos \phi_{g}}+\frac{o}{b_{g} \sin \phi_{g}}\right] \sin n \mu_{x} \\
& \equiv r \cos n \mu_{x}+s \sin n \mu_{x}
\end{aligned}
$$


Similarly we find:

$$
\begin{aligned}
p_{y_{n, g}}^{U} & =\left[\frac{d_{g} p}{b_{g} \cos \phi_{g}}-\frac{l}{b_{g} \sin \phi_{g}}\right] \cos n \mu_{y}+\left[\frac{d_{g} q}{b_{g} \cos \phi_{g}}-\frac{m}{b_{g} \sin \phi_{g}}\right] \sin n \mu_{y} \\
& \equiv t \cos n \mu_{x}+u \sin n \mu_{x}
\end{aligned}
$$

From $x_{n, g}^{U}$ and $p_{x n, g}^{U}$ we obtain $A_{x g, A}$ and $R_{x g, A}$. From $y_{n, g}^{U}$ and $p_{y_{n, g}}^{U}$ we obtain $A_{y g, A}$ and $R_{y g, A}$. Now we can construct the entire guess matrix.

$$
\begin{aligned}
G_{A, A} & \equiv G_{A, A}\left(g_{x, g}, \theta_{x y, g}, g_{y, g}, \theta_{y x, g}, a_{g}, b_{g}, c_{g}, \phi_{g}\right) \\
& =C_{A, g} A_{A, g} R_{A, G} A_{A, g}^{-1} C_{A, g}^{-1}
\end{aligned}
$$

With the initial condition $\overrightarrow{x_{0, g}}=C_{A, g}\left(x_{0, g}^{U}, p_{x, g}^{U}, y_{0, g}^{U}, p_{y_{0, g}}^{U}\right)^{T}$ we see by working backwards from Eq.(90) and Eq.(91) that the symplectic matrices $G_{A, A}$, given by Eq.(92), will all produce the same $x_{n}$ and $y_{n}$ orbits as those in the observed orbit data set. As 8 parameters are randomly chosen to obtain the $G_{A, A}$, the $x$-orbit data from a single $B P M$ alone must be insufficient to determine the 1-turn map $M_{A, A}$. Further we must have $\mathcal{D}=8$ exactly as all the remaining $14-8=6$ parameters are uniquely determined once these 8 are specified.

\subsection{Multiple BPM Analysis}

We begin by considering a 2-Dimensional lattice consisting of 2 BPMs. Again we shall show that the constraints supplied by the observable data are insufficient to determine the matrices $M_{A, A}$, and $M_{B, A}$. We shall then extend this result to the general storage ring consisting of $N B P M \mathrm{~s}$.

\section{Number of Independent Orbits}

To show that we need only consider a single orbit data set we may use the same argument as was used in the 1-dimensional lattice case.

Consider two orbit data sets, each consisting of the $x$ and $y$ data from $B P M_{A}$ and $B P M_{B}$ :

$$
\text { set1 : }\left(\overrightarrow{x_{0, A}}, \overrightarrow{x_{1, A}}, \ldots ; \overrightarrow{x_{0, B}}, \overrightarrow{x_{1, B}}, \ldots\right) \quad \operatorname{set} 2:\left(\overrightarrow{X_{0, A}}, \overrightarrow{X_{1, A}}, \ldots ; \overrightarrow{X_{0, B}}, \overrightarrow{X_{1, B}}, \ldots\right)
$$

Looking first at only the data from $B P M_{A}$ we may normalize the second orbit data set, obtaining a new one, $\left(\overrightarrow{X_{0, A}^{\star}}, \overrightarrow{X_{1, A}^{\star}}, \ldots, \overrightarrow{X_{0, B}^{\star}}, \overrightarrow{X_{1, B}^{\star}}, \ldots\right)$, which is contained in the first:

$$
\overrightarrow{x_{j(k), A}}, \overrightarrow{x_{j(k)+1, A}}, \overrightarrow{x_{j(k)+2, A}}, \ldots \simeq \overrightarrow{X_{0, A}^{\star}}, \overrightarrow{X_{1, A}^{\star}}, \overrightarrow{X_{2, A}^{\star}}, \ldots
$$

as in the above. Now multiplying on the left by $M_{B, A}$ we obtain:

$$
\begin{array}{r}
M_{B, A} \overrightarrow{x_{j(k), A}}, M_{B, A} \overrightarrow{x_{j(k)+1, A}}, \ldots=\overrightarrow{x_{j(k), B}}, \overrightarrow{x_{j(k)+1, B}}, \ldots \\
\simeq M_{B, A} \overrightarrow{X_{0, A}^{\star}}, M_{B, A} \overrightarrow{X_{1, A}^{\star}}, \ldots=\overrightarrow{X_{0, B}^{\star}}, \overrightarrow{X_{1, B}^{\star}}, \ldots \rightarrow \\
\overrightarrow{x_{j(k), B}}, \overrightarrow{x_{j(k)+1, B}}, \overrightarrow{x_{j(k)+2, B}}, \ldots \simeq \overrightarrow{X_{0, B}^{\star}}, \overrightarrow{X_{1, B}^{\star}}, \overrightarrow{X_{2, B}^{\star}}, \ldots
\end{array}
$$


showing that the 2 nd orbit is contained in the first at $B P M_{B}$ as well. This argument can be extended to any number of $B P M$ s by proceeding inductively. In general we see that for 2-dimensional lattices it is possible to normalize an orbit data set so that it will be contained in another. As a single orbit data set contains all others (up to this normalization), this implies we need only consider one when attempting to determine the matrices $M_{A, A}, M_{B, A}, M_{C, B}$, etc..

\section{Construction of Guess Matrices}

Consider a single orbit data set $\overrightarrow{x_{A 0, R}}, \overrightarrow{x_{A 1, R}}, \ldots, \overrightarrow{x_{B 0, R}}, \overrightarrow{x_{B 1, R}}, \ldots$ Using only the data from $B P M_{A}$ we could proceed as in the above and find a family of matrices which could produce the observed data at $B P M_{A}$. Similarly using the data from $B P M_{B}$ we could find another family of matrices which produce the observed data at $B P M_{B}$. For each choice of the symplectic guess matrices $G_{A, A}$ and $G_{B, B}$ we obtain a different guess at the actual phase space orbits of the beam and thus a different guess matrix $G_{B, A}$,

$$
G_{B, A}=\left(\overrightarrow{x_{B, 0}}, \overrightarrow{x_{B, 1}}, \overrightarrow{x_{B, 2}}, \overrightarrow{x_{B, 3}}\right)\left(\overrightarrow{x_{A, 0}}, \overrightarrow{x_{A, 1}}, \overrightarrow{x_{A, 2}}, \overrightarrow{x_{A, 3}}\right)^{-1}
$$

(the fact that $G_{B, A}$ will map the entire orbit at $A$ to the orbit at $B$ can be shown easily, by considering the map in the uncoupled space.) We must apply the symplectic condition to $G_{B, A}$ to determine which of the $G_{A, A}$ and $G_{B, B}$ are valid guesses. We may simplify the application of the symplectic condition to these matrices by first factoring them. Note that we can decompose the $G_{B, A}$ in the following form,

$$
G_{B, A}=\widetilde{A}_{B, g} U \widetilde{A}_{A, g}^{-1}=C_{B, g} A_{B, g} U A_{A, g}^{-1} C_{A, g}^{-1}
$$

for some matrix $U$ as the matrices $A_{g}$ and $C_{g}$ are all invertible. By definition:

$$
\begin{aligned}
G_{A, A} & =G_{B, A}^{-1} G_{B, B} G_{B, A} \rightarrow \\
C_{A, g} A_{A, g} R_{A, A} A_{A, g}^{-1} C_{A, g}^{-1} & =G_{B, A}^{-1} C_{B, g} A_{B, g} R_{B, B} A_{B, g}^{-1} C_{B, g}^{-1} G_{B, A}
\end{aligned}
$$

Plugging in Eq.(96) we get:

$$
R_{A, A}=U^{-1} R_{B, B} U
$$

The rotations $R_{A, A}$ and $R_{B, B}$ share the same 4 eigenvectors, namely: $(1, \pm \imath, 0,0)^{T}$ and $(0,0,1, \pm \imath)^{T}$. As these matrices are similar under $U$ it follows that $U$ maps this set of eigenvectors to itself modulo some scalar factors. Further, as $U$ is real, it maps complex conjugate vectors to complex conjugate vectors. Therefore either $U(1, \pm \imath, 0,0)^{T}=a(1, \pm \imath, 0,0)^{T}$ or $U(1, \pm \imath, 0,0)^{T}=a(0,0,1, \pm \imath)^{T}$. However, we know that $G_{B, A}$ will not swap the $x-p_{x}$ and $y-p_{y}$ eigenplanes implying that $U(1, \pm \imath, 0,0)^{T}=a(1, \pm \imath, 0,0)^{T}$ and similarly $U(0,0,1, \pm \imath)^{T}=$ $b(0,0,1, \pm \imath)^{T}$ so that $U$ is block diagonal.

$$
U=\left(\begin{array}{cc}
U_{x} & 0_{2 x 2} \\
0_{2 x 2} & U_{y}
\end{array}\right)
$$


We may now easily apply the symplectic condition to the matrices $G_{B, A}$. The $C_{g}$ and $A_{g}$ matrices are symplectic by construction. Therefore by Eq.(96), requiring $G_{B, A}$ symplectic is equivalent to requiring the block diagonal matrix $U_{g}$ to be symplectic. Plugging in Eq.(99) to Eq.(55), we see this is equivalent to requiring each of the diagonal matrices of $U$ to be symplectic. Thus we get the following two symplectic constraints:

$$
\begin{aligned}
& \operatorname{det} U_{g, x}=\operatorname{det}\left(\begin{array}{cc}
x_{0, g} & x_{1, g} \\
p_{x 0, g} & p_{x 1, g}
\end{array}\right)_{B}\left(\begin{array}{cc}
x_{0, g} & x_{1, g} \\
p_{x 0, g} & p_{x 1, g}
\end{array}\right)_{A}^{-1}=1 \\
& \operatorname{det} U_{g, y}=\operatorname{det}\left(\begin{array}{cc}
y_{0, g} & y_{1, g} \\
p_{y 0, g} & p_{y 1, g}
\end{array}\right)_{B}\left(\begin{array}{cc}
y_{0, g} & y_{1, g} \\
p_{y 0, g} & p_{y 1, g}
\end{array}\right)_{A}^{-1}=1
\end{aligned}
$$

Applying these constraints we obtain the symplectic matrices $G_{A, A}$ and $G_{B, B}$ which are consistent with a symplectic map $G_{B, A}$ and produce the observed orbit data. As 14 parameters are randomly chosen to obtain these matrices ( 8 from each of the two 1-turn maps, minus 2 due to the symplectic conditions, Eq.(100) and Eq.(101)), the $x$-orbit data from $2 B P M$ s alone must be insufficient to determine the maps $M_{A, A}$ and $M_{B, A}$. Further $\mathcal{D}=14$ exactly as the remaining $28-14=14$ parameters are uniquely determined once these 14 are chosen.

\section{Extension to Multiple BPMs}

The extension to a lattice with $N B P M$ s follows very easily. By the above we need only consider a single orbit data set,

$$
\overrightarrow{x_{A 0, R}}, \overrightarrow{x_{A 1, R}}, \ldots ; \overrightarrow{x_{B 0, R}}, \overrightarrow{x_{B 1, R}}, \ldots ; \ldots ; \overrightarrow{x_{N 0, R}}, \overrightarrow{x_{N 1, R}}, \ldots
$$

when attempting to determine the matrices $M_{A, A}, M_{B, A}, \ldots, M_{N, N-1}$. We may construct with 8 degrees of freedom each, the matrices $G_{A, A}, G_{B, B}, \ldots$, and $G_{N, N}$. Applying the $2(N-1)$ symplectic constraint equations similar to Eq.(100) and Eq.(101) on the matrices $G_{B, A}, G_{C, B}, \ldots, G_{N, N-1}$, we are left with the infinite set of matrices $G_{A, A}, \ldots, G_{N, N}$, which are consistently symplectic and produce the observed $x_{n}$ and $y_{n}$ orbit data. The constraints supplied by the observable data are again insufficient to determine the matrices $M_{A, A}, M_{B, A}$, $\ldots, M_{N, N-1}$ and leave the global number of undetermined parameters in these matrices at $\mathcal{D}=8 \mathrm{~N}-2(\mathrm{~N}-1)=6 \mathrm{~N}+2$.

\subsection{Approximate Solution and Future Work}

For a 2-dimensional lattice consisting of $N B P M \mathrm{~s}$, we saw above that the observable data supplies an insufficient number of constraints to specify correctly the matrices $M_{A, A}, M_{B, A}, \ldots, M_{N, N-1}$. We must therefore turn to the method described in section 2.4 to determine these matrices.

Suppose we have $x$ and $y$-orbit data from both $B P M_{A}$ and $B P M_{B}$ and further that we have knowledge of the symplectic matrix $M_{B, A}$. As there are 10 independent elements in a $4 x 4$ symplectic matrix, knowledge of $M_{B, A}$ supplies 
10 constraint equations. However, the system is still under-constrained as the observable data leaves the number of unknown parameters at $\mathcal{D}=14$. Therefore, even if $M_{B, A}$ is known, we still cannot determine $M_{A, A}$; a result which differs from its 1-dimensional analog.

If we have $x$ and $y$-orbit data from $3 B P M$ s and knowledge of both $M_{B, A}$ and $M_{C, B}$ it may then be possible to determine the matrix $M_{A, A}$ : The observable data leaves the number of unknown parameters at $\mathcal{D}=20$ but we also obtain 20 additional constraint equations from knowledge of $M_{B, A}$ and $M_{C, B}$. Although it may be theoretically possible to determine $M_{A, A}$ using just $3 B P M \mathrm{~s}$, an overconstrained system would be preferable as the use of some least squares fitting approach would enable the reduction of error. Therefore we have proven that 3 is a lower bound on the number of $B P M$ s required to have a properly constrained system while we recommend 4 as the minimum to be used in practice.

The use of $4 B P M$ s will soon be tested numerically here at SLAC. Pending those results, 2 new $B P M$ s may be installed near the interaction point of the LER and HER rings, in the hope that using a localized measurement approach in conjunction with the current global scheme may improve beam position measurements and ultimately increase the efficiency of the machine.

\section{Acknowledgements}

I would like to thank my advisor, Dr. Yiton Yan, who has been an excellent mentor, Dr. James Lindesay and Dr. Helen Quinn, the directors of the SULI program here at SLAC, Deji Akinwande, Dr. Tom Knight, and William Colocho, who have all helped me in various ways this summer, and lastly, the United States Department of Energy - Office of Science for providing funding towards what has been for me a very rewarding and educational summer internship. 


\section{References}

[1] Y. T. Yan, Y. Cai, F-J. Decker, et. al., Virtual Accelerator for Accelerator Optics Improvement. SLAC-PUB-11209, May 2005

[2] Robert L. Devaney, An Introduction to Chaotic Dynamical Systems. Westview Press, Massachusetts, 2nd Edition, 2003.

[3] Philip Tanedo, Modeling of the Transverse Linear Optics of a Charged Particle Storage Ring. SLAC-TN-03-016, Sep 2003.

[4] Y. T. Yan, The Use of Lie Algebra Methods to Analyze and Design Accelerator Beamlines. Notes from the U.S. Particle Accelerator School; Rice University, Houston, Texas. January, 2001. 